\title{
Otimização construtal de uma cavidade multi-bifurcada para dissipação de calor
}

\author{
Constructal optimization of a multi-forked cavity for heat dissipation \\ R. A. Gouvêa; E. D. Santos, J. A. Souza* \\ FURG, Avenida Itália km 8, Campus Carreiros, Rio Grande, RS, 96201-900, Brazil. \\ *jasouza1974@gmail.com
}

(Recebido em 29 de abril de 2017; aceito em 22 de maio de 2017)

\begin{abstract}
O propósito do trabalho é analisar a influência dos parâmetros geométricos sobre a dissipação de calor de um perfil bidimensional com uma cavidade multi-bifurcada mantendo a área da cavidade constante. A simulação numérica foi realizada através da solução da equação da difusão do calor com MEF. Foi calculado o campo de distribuição de temperaturas no regime permanente e com geração de calor interna fixa e foi assumido que a transferência de calor no sólido ocorreu por condução. A geometria da cavidade foi gerada por um sistema de funções iteradas e um algoritmo foi implementado para testar diferentes ângulos de bifurcação, tamanho de cada braço de ramificação e número de iterações para geração da geometria. Entre as cavidades testadas foi possível determinar uma geometria ótima que serve de recomendação teórica para construção de possíveis perfis para troca de calor.
\end{abstract}

Palavras-chave: MEF, dissipação de calor, design construtal.

The purpose of this work is to analyze the influence of geometric parameters on the heat dissipation of a two-dimensional profile with a multi-bifurcated cavity keeping the cavity area constant. The numerical simulation was performed through the solution of the heat diffusion equation with MEF. The temperature distribution field was calculated in the steady state and with fixed internal heat generation and it was assumed that heat transfer to the solid occurred by conduction. The cavity geometry was generated by a system of iterated functions and an algorithm was implemented to test different bifurcation angles, size of each branching arm and number of iterations for geometry generation. Among the wells tested, it was possible to determine an optimum geometry that serves as a theoretical recommendation for the construction of possible profiles for heat exchange.

Keywords: FEM, heat dissipation, constructal design.

\section{INTRODUÇÃO}

Fractais tem sido usados em diversas áreas de engenharia e o potencial dessa teoria começa a ser importante em diversas aplicações industriais reais [1]. O uso da teoria dos fractais abrange aplicações na área de física [2, 3], engenharia química [4, 5], finanças [6, 5], tráfego de internet [7], processamento de imagens [8,9], dinâmica dos fluidos [10, 11], ciência ambiental [12, 13], análise de superfícies [14] dentre outros. O motivo dessa gama de aplicações da teoria fractal se deve particularmente a sua característica de descrever a auto-similaridade em diferentes escalas que é uma aproximação do que ocorre na natureza $[15,16]$. Com uma formulação mais prática, existe também a Teoria Construtal que procura explicar deterministicamente o surgimento de formas na natureza a partir da ideia de um sistema de fluxo que altera sua forma para facilitar o acesso das correntes que fluem através dele. Dentro da Teoria Construtal é definido por Adrian Bejan o método Design Construtal como o uso da Lei Construtal como uma ferramenta na procura de melhores configurações e também melhores estratégias para resolução de um determinado problema que depende de geometria [17].

Um problema frequente de engenharia que envolve geometria é a transferência de calor em um corpo sólido. Como no caso de reatores nucleares e micro-eletrônica, o modelo mais aplicado é aquele de um corpo contendo uma cavidade. Nas cavidades do corpo é onde a troca de calor entre o fluído de resfriamento e o sólido é mais intensa, como ocorre no caso do efeito Vapotron [18]. Com o desenvolvimento tecnológico o requerimento de melhor dissipação do calor tem 
aumentado sendo crucial otimizar o projeto dos diversos modelos de cavidade a fim de melhorar a performance do sistema.

De acordo com Bejan (1997) [19], chega um ponto onde a miniaturização torna o resfriamento por convecção impraticável, porque os dutos nos quais o fluído de resfriamento deve passar começam a tomar muito espaço. A única maneira eficiente de canalizar o calor gerado para fora do sistema é por condução. Esse caminho de condução terá de ser muito efetivo (alta condutividade térmica, $\mathrm{k} \mathrm{p}$ ), de maneira que a diferença de temperatura entre o ponto mais quente e o dissipador de calor não exceda um determinado valor. A partir dessa constatação, diversos estudos vêm sendo realizados avaliando diferentes formas geométricas para cavidade de dissipação de calor com Design Construtal a fim de encontrar arranjos ótimos para aumentar a troca térmica dentro das restrições impostas. Podemos citar os trabalhos em cavidade em forma de 'C' [20,21], 'T' [22], 'H' [23] e até mesmo formas mistas como 'T-Y' [24].

Neste trabalho o problema de dissipação de calor em uma cavidade é tratado. Inicialmente é definido o espaço de busca apropriado para as geometrias de cavidade aplicando o Design Construtal, logo utilizando como base geometrias geradas por um sistema de função iterada (SFI), que é uma técnica de geração de fractais, são geradas cavidades que são testadas quanto a eficiência na dissipação de calor. A equação do calor com geração interna de energia foi resolvida para diferentes variações dessa geometria de forma a determinar os parâmetros geradores do SFI que otimizam a dissipação do calor no sistema. Ao final, os parametros do sistema de função iterada determinando a forma geométrica que leva a temperatura máxima do sistema a um mínimo foi escolhida como ótima. O efeito de cada parâmetro de geração da geometria do SFI na temperatura máxima do sistema também foi estudado.

Apesar da diferença conceitual entre Teoria Fractal e a Lei Construtal, esse trabalho parte do conceito de Design Construtal que permite a procura de melhores estratégias e não apenas configurações para resolução de um problema geométrico. Dessa forma, o sistema de funções iteradas, e portanto a Teoria Fractal, serve apenas como uma estratégia de busca para as melhores geometrias que resolvam o problema da cavidade similar a aplicação de metaheurísticas como algoritmo genético e recozimento simulado. A principal vantagem do uso desse método reside na possibilidade de vasculhar o espaço de busca com uma geometria mais apropriada ao problema que, como a própria Lei Construtal aponta, é geralmente uma forma que apresenta autosimilaridade em alguma escala [25].

\section{METODOLOGIA}

Toda a implementação deste trabalho foi realizada com auxílio do software comercial MATLAB [26]. As etapas foram divididas em: geração da geometria da cavidade através de função iterada, criação do domínio computacional com a cavidade, geração de malha, solução da equação do calor para o sistema. O fluxograma do algoritmo para realizar a simulação é mostrado na Fig. 1. O detalhamento de cada etapa será apresentado nas subseções a seguir. 


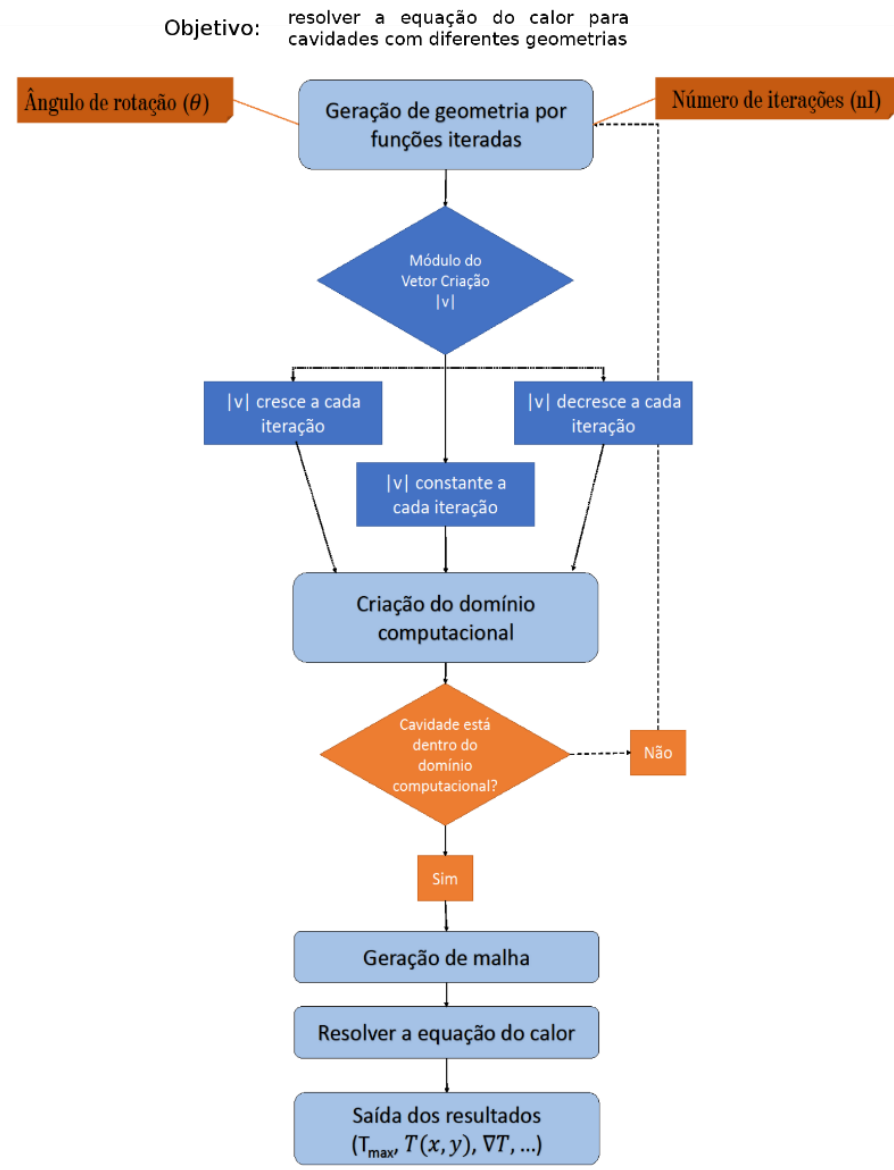

Figura 1: Fluxograma do algoritmo implementado nesse trabalho.

\subsection{EQUAÇÕES FUNDAMENTAIS}

Neste trabalho aplicou-se uma forma simplificada da equação da difusão do calor pois foi realizada uma análise bidimensional de uma cavidade em uma placa de material homogêneo em regime permanente. A equação da difusão do calor para condução para o caso em estudo é dada por:

$\frac{\partial^{2} T}{\partial x^{2}}+\frac{\partial^{2} T}{\partial y^{2}}+\frac{q^{\prime \prime \prime}}{k}=0$

onde $(\mathrm{x}, \mathrm{y})$ determina um ponto no domínio computacional, $\mathrm{T}=\mathrm{T}(\mathrm{x}, \mathrm{y})$ é a temperatura absoluta em um determinado ponto, q" é a geração interna de calor por volume $\left(\mathrm{W} \cdot \mathrm{m}^{-2}\right)$ e as propriedades do meio ficam especificadas pela condutividade térmica $\mathrm{k}=\mathrm{k}(\mathrm{x}, \mathrm{y}) \mathrm{em} \mathrm{W} \cdot \mathrm{K}^{-1}$.

\subsection{MÉTODO DE ELEMENTOS FINITOS E ANÁLISE DE ERRO}

O método de elementos finitos (MEF) é uma técnica numérica para encontrar soluções aproximadas de problemas de contorno para equações diferenciais parciais (EDP) $[27,28]$. O problema maior de encontrar a solução da EDP fica subdividido em partes menores e mais simples chamadas elementos finitos. Para cada sub-domínio (elemento) é escrita uma equação algébrica obtida a partir da interpolação da EDP original essas equações algébricas são então montadas num sistema maior de equações que modela o problema por inteiro. Esse conjunto de equações é resolvido por métodos variacionais para aproximar uma solução minimizando a função de erro. Alguns pontos importantes do MEF são citados abaixo [29]: 
(a) Através do uso de funções interpoladoras a solução é aproximada para qualquer ponto do domínio; (b) A equação é resolvida na forma integral, ou seja, a equação diferencial é satisfeita segundo um certo critério de minimização do resíduo. Isto resulta em mais flexibilidade e generalidade no método para resolver equações diferenciais; (c) Os sistemas matriciais obtidos são em geral simétricos e esparsos, não gerando grande custo na sua solução; (d) Apesar da implementação complexa o MEF é genérico sendo facilmente aplicado em domínios irregulares.

Visto a generalidade e praticidade do MEF este foi usado conforme implementado no pacote PDE TOOL do MATLAB que usa um algoritmo de refinamento de malha adaptável e transforma a EDP genérica mostrada na Eq. 2 para o cálculo de problemas de autovalores:

$$
\nabla(c \nabla u)+a u=\lambda d u
$$

na seguinte forma matricial equivalente:

$$
\mathbf{K U}=\lambda \mathbf{M U}
$$

onde $\mathrm{M}$ é a matriz de massa e $\mathrm{K}$ é conhecida como a matriz de rigidez. Essa equação de autovalores é então resolvida por um algoritmo de Arnoldi até todos autovalores serem encontrados para um determinado intervalo. O algoritmo de Arnoldi passa por iterações de GramSchmidt para achar vetores ortonormais do subespaço de Krylov. Sendo o problema discretizado e interpolado por funções lineares existe um erro inerente da técnica que precisa ser levado em consideração.

A fim de estimar o erro associado ao cálculo por MEF no PDE TOOL foi considerado um problema onde a solução analítica é conhecida, um domínio retangular com condições de Dirichlet $(\mathrm{T}=0 \mathrm{~K})$ em uma face enquanto as outras faces são mantidas adiabáticas por condições de Neumann. Esse sistema e suas condições de contorno são mostradas na Fig. 2:

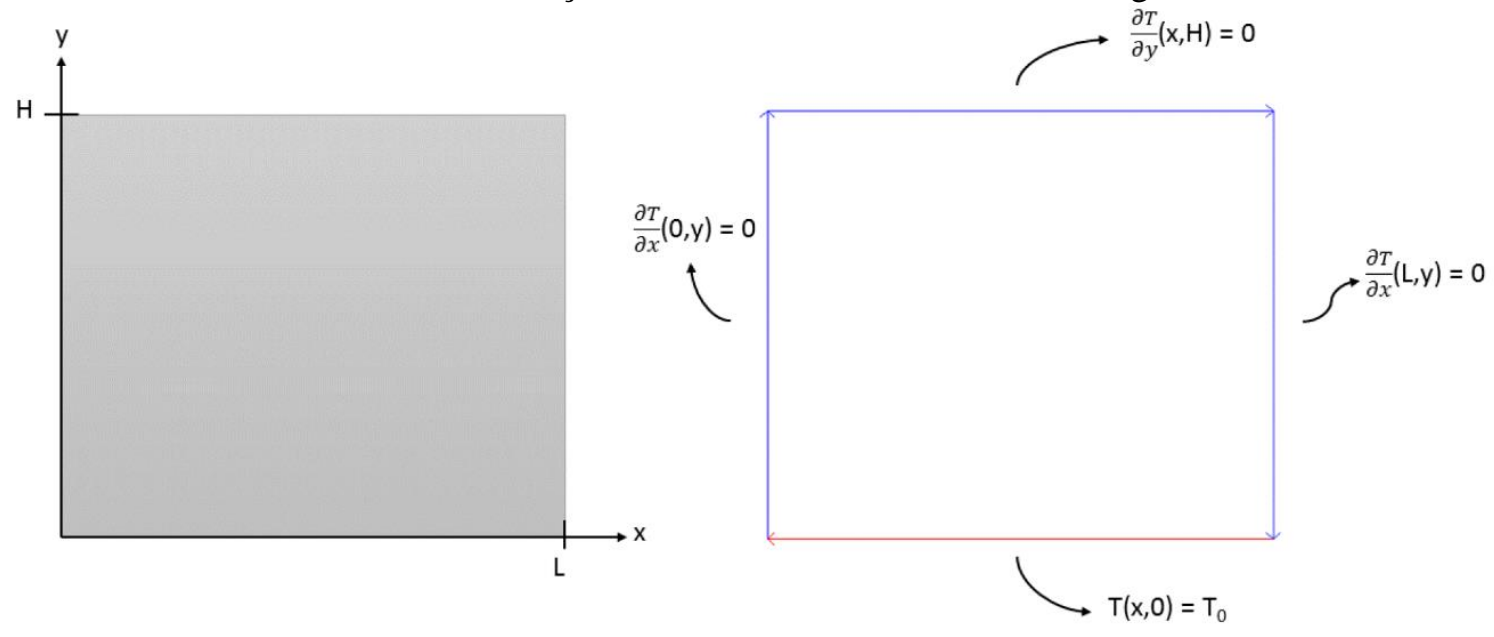

Figura 2: Domínio simples para resolução analítica.

Considerando a equação do calor para esse caso temos:

$$
\frac{\partial^{2} T}{\partial x^{2}}+\frac{\partial^{2} T}{\partial y^{2}}+\frac{q^{\prime \prime \prime}}{k}=0 \quad T(x, 0)=\left.T_{0} \quad \frac{\partial T}{\partial x}\right|_{(0, y)}=\left.0 \quad \frac{\partial T}{\partial x}\right|_{(L, y)}=\left.0 \quad \frac{\partial T}{\partial y}\right|_{(x, H)}=0
$$

Fazendo a seguinte troca de variável para tornar as condições de contorno homogêneas:

$$
\vartheta=T-T_{0}
$$

É possível reescrever a Eq. 4 como:

$$
\frac{\partial^{2} \vartheta}{\partial x^{2}}+\frac{\partial^{2} \vartheta}{\partial y^{2}}+\frac{q^{\prime \prime \prime}}{k}=0 \quad \vartheta(x, 0)=\left.0 \quad \frac{\partial \vartheta}{\partial x}\right|_{(0, y)}=\left.0 \quad \frac{\partial \vartheta}{\partial x}\right|_{(L, y)}=\left.0 \quad \frac{\partial \vartheta}{\partial y}\right|_{(x, H)}=0
$$


(6)

A equação diferencial não é homogênea mas agora é possível desmembrar $\vartheta$ em uma solução particular da EDP não-homogênea adicionada a solução homogênea:

$$
\vartheta(x, y)=\phi(y)+\psi(x, y)
$$

Colocando a particular assim sugerida da EDP recai na seguinte EDO:

$$
\frac{d^{2} \phi}{d x^{2}}+\frac{q^{\prime \prime \prime}}{k}=0,\left.\quad \frac{d \phi}{d y}\right|_{H}=0 \quad e \quad \phi(0)=0
$$

Essa EDO é resolvida por integração e aplicação das condições de contorno resulta em:

$$
\phi=-\frac{q^{\prime \prime \prime}}{2 k} y^{2}+\frac{q^{\prime \prime \prime}}{k} H . y
$$

A equação homogênea assume a forma:

$$
\frac{\partial^{2} \psi}{\partial x^{2}}+\frac{\partial^{2} \psi}{\partial y^{2}}+\frac{q^{\prime \prime \prime}}{k}=0 \quad \psi(x, 0)=\left.0 \quad \frac{\partial \psi}{\partial x}\right|_{(0, y)}=\left.0 \quad \frac{\partial \psi}{\partial x}\right|_{(L, y)}=0 \quad\left[\left.\frac{\partial \psi}{\partial y}\right|_{(x, H)}=0\right.
$$

Obviamente a única solução da homogênea será a solução trivial $\psi(\mathrm{x}, \mathrm{y})=0$, logo a solução geral da EDP pela Eq. 7, para as condições de contorno apresentadas é:

$$
\vartheta(x, y)=-\frac{q^{\prime \prime \prime}}{2 k} y^{2}+\frac{q^{\prime \prime \prime}}{k} H \cdot y
$$

Se $\mathrm{T}_{0}=0$ então $\vartheta(\mathrm{x}, \mathrm{y})=\mathrm{T}(\mathrm{x}, \mathrm{y})$, e $\mathrm{T}_{\max }$ para esse sistema será:

$$
T_{\max }=-\frac{q^{\prime \prime \prime}}{2 k} H^{2}+\frac{q^{\prime \prime \prime}}{k} H . H=\frac{q^{\prime \prime \prime}}{2 k} H^{2}
$$

Essa geometria foi gerada no PDE TOOL afim de verificar a concordância com a resposta analítica. Os parâmetros foram $\mathrm{k}=1 \mathrm{~W} \cdot \mathrm{m}^{-3}, \mathrm{q}$ "' $=1 \mathrm{~W} \cdot \mathrm{m}^{-1} \cdot \mathrm{K}^{-1}$ e $\mathrm{H}=1 \mathrm{~m}$, de forma que a resposta analítica resulta em um $\mathrm{T}_{\max }=0,5$. A Tab. 1 mostra o erro do $\mathrm{T}_{\max }$ dado pela Eq. 13, $\mathrm{T}_{\max , \mathrm{i}}$ é valor obtido da temperatura máxima pelo PDE TOOL conforme o número de vezes que a malha foi refinada e $\mathrm{T}_{\max , \mathrm{o}}$ é o valor da temperatura máxima obtido teoricamente para $\mathrm{o}$ problema.

Erro $_{T \max }=\frac{T_{\max , i}-T_{\max , o}}{T_{\max , o}}$

Tabela 1: Comparação do número de refinamentos de malha no PDE TOOL com erro do $T_{\max }$ obtido em relação à resposta analítica.

\begin{tabular}{ccc}
\hline Número de refinamentos & Número de Nós & Erro de T max \\
\hline 1 & 177 & $5,138754.10^{-4}$ \\
2 & 665 & $1,841925.10^{-4}$ \\
3 & 2577 & $5,993222.10^{-5}$ \\
4 & 10145 & $1,844573.10^{-5}$ \\
\hline
\end{tabular}

Um critério de erro $<0,0002$ já é suficiente para o presente estudo, por esse motivo, apenas 2 refinamentos de malha serão utilizados para resolver a EDP por elementos finitos no PDE TOOL. 


\subsection{SISTEMA DE FUNÇÕES ITERADAS E GEOMETRIA DA CAVIDADE}

Sistemas de funções iteradas (SFI) é um método de construir fractais que consiste da união de sucessivas cópias da forma inicial cada uma delas sendo transformada por uma função. Esses sistemas foram concebidos na forma atual por John E. Hutchinson em 1981 e popularizados no livro Fractals Everywhere de Michael Barnsley [30]. Bransley destaca que "Sistemas de funções iteradas fornecem um modelo descritivo para certas plantas, folhas e árvores pelo fator de autosimilaridade que ocorrem com frequência em estruturas ramificadas na natureza". Alguns exemplos de fractais famosos obtidos por esse método como o tapete de Sierpinski e a Árvore Simétrica são mostrados na Fig. 3. Nesse sentido, as geometrias obtidas através da geometria fractal, apesar de consistirem basicamente de um modelo descritivo para as formas da natureza, podem servir de um modelo para avaliar o espaço de busca definido na aplicação do Design Construtal. Uma vez que o Design Construtal prevê que geometrias ramificadas tendem a diminuir a resistência ao fluxo em muitos casos. Baseando-se nessa ideia, esse trabalho gerou a cavidade para a um dissipador de calor usando um sistema de funções iteradas que resulta em uma forma de árvore. Esse procedimento apresenta algumas vantagens em relação a uma geometria fixa como vêm sendo estudado na literatura com cavidades em forma de ' $T$ ', ' $\mathrm{H}$ ', ' $\mathrm{Y}$ ' e etc. Primeiramente, o SFI permite controlar facilmente as geometrias resultantes ajustando poucos parâmetros, inclusive as geometrias fixas referidas anteriormente podem ser obtidas por um mesmo sistema de funções iteradas. Em segundo lugar, abordando a geração de geometria por um SFI torna mais amplo o estudo dos graus de liberdade do sistema variando parâmetros na lei de geração do fractal ao invés de estudar cada razão geométrica isoladamente. Além disso, pela Teoria Fractal já ser um campo de pesquisa consolidado o uso de certas ferramentas como autosimilaridade, dimensão fractal e número de iterações pode ser reaproveitado no estudo da geometria do sistema.

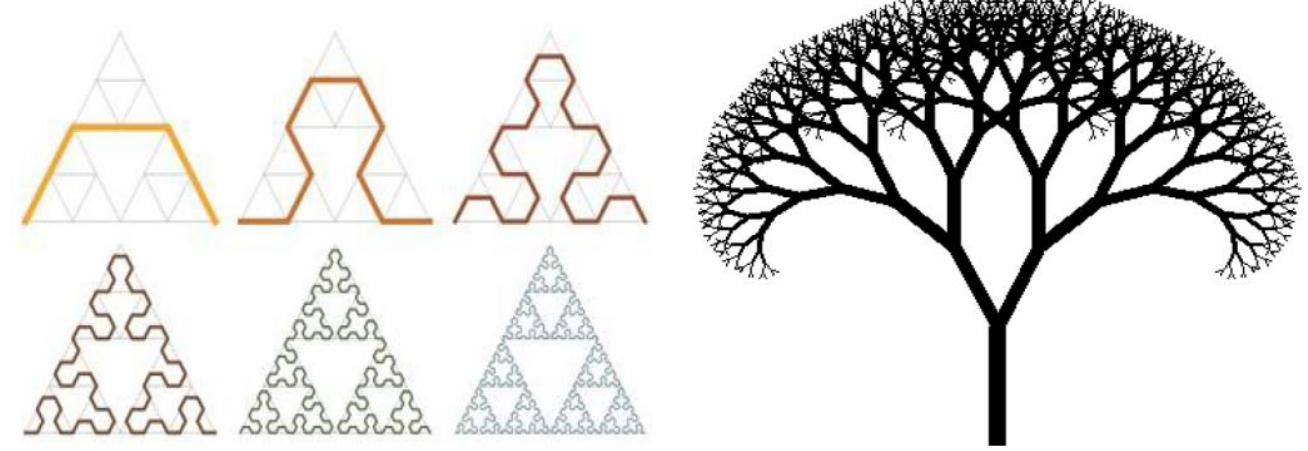

Figura 3: Fractais famosos obtidos por sistemas de funções iteradas (SFI). Fonte: http://fractalfoundation.org/.

A forma da cavidade escolhida se baseou numa árvore simétrica binária, inicialmente com escalamento $r=1$, isto é, o tamanho de cada galho permanece o mesmo em sucessivas iterações do SFI. As equações para esse sistema são mostradas abaixo:

$$
\begin{aligned}
& f_{1}(\mathbf{x})=\left[\begin{array}{cc}
r \cdot \cos (\theta) & -r \cdot \sin (\theta) \\
r \cdot \sin (\theta) & r \cdot \cos (\theta)
\end{array}\right] \mathbf{x}+\left[\begin{array}{l}
0 \\
1
\end{array}\right] \\
& f_{2}(\mathbf{x})=\left[\begin{array}{cc}
r \cdot \cos (\theta) & r \cdot \sin (\theta) \\
-r \cdot \sin (\theta) & r \cdot \cos (\theta)
\end{array}\right] \mathbf{x}+\left[\begin{array}{l}
0 \\
1
\end{array}\right]
\end{aligned}
$$


Essas equações correspondem a uma rotação em sentido horário e anti-horário de um ponto seguido de uma translação. Elas são aplicadas sucessivamente em um algoritmo implementado no MATLAB gerando $2^{\mathrm{nI}}$ novos pontos na estrutura onde $\mathrm{nI}$ é o número de iterações que formarão a geometria, ou seja, o nI está diretamente relacionado ao número de braços da cavidade resultante. Um exemplo ilustrativo juntamente com algumas geometrias calculadas é mostrado na Fig. 4.

Para gerar as estruturas é preciso fornecer o módulo do vetor de criação $|\mathrm{v}|$ (corresponde ao tamanho do braço), o ângulo de rotação $(\theta)$ que será igual as duas direções e o número de iterações, isto é, quantas vezes $\mathrm{f}_{1}$ e $\mathrm{f}_{2}$ serão aplicadas na geometria.

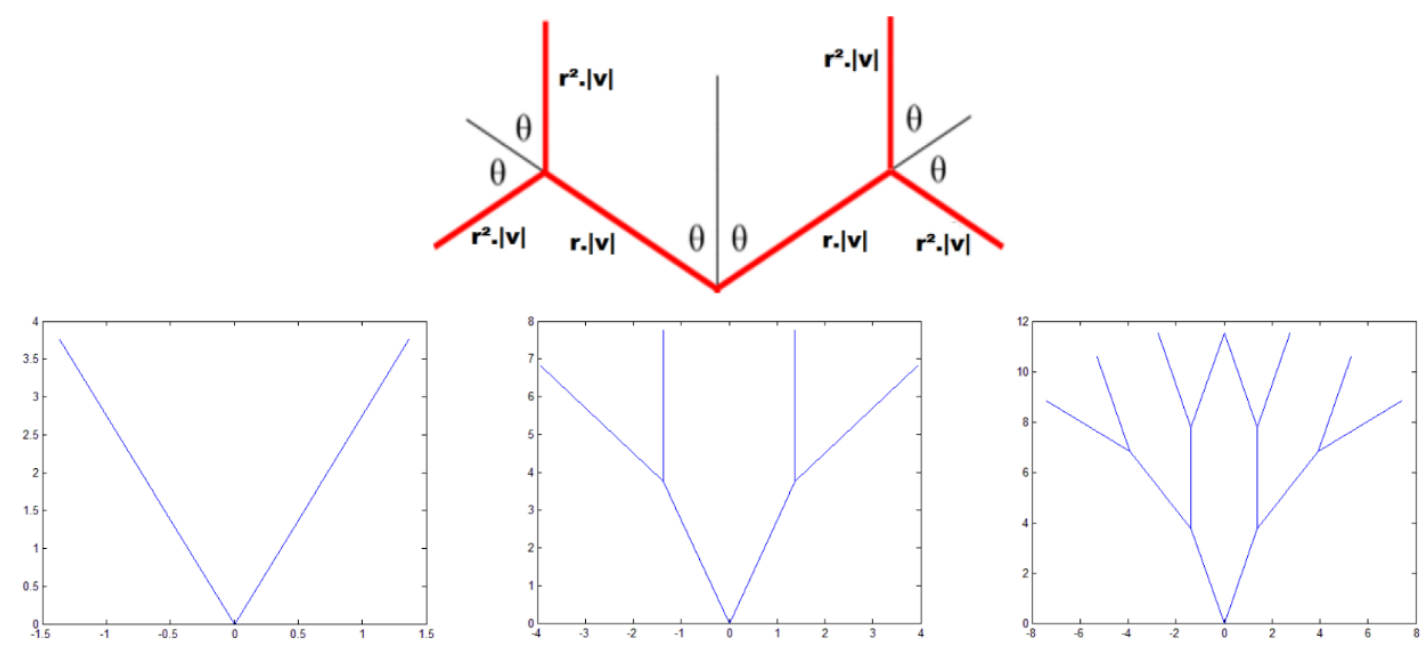

Figura 4: Ilustração da geração do fractal assim como algumas formas de cavidade calculadas.

\subsection{REPRESENTAÇÃO COMPUTACIONAL}

Para transformar a geometria obtida anteriormente em uma cavidade que possibilite resolver a EDP do calor dentro do PDE TOOL, é preciso adicionar espessura a geometria do SFI e então subtraí-la do domínio computacional de interesse dentro do PDE TOOL. O domínio computacional inicial foi um retângulo $1 \mathrm{~m}$ x $1 \mathrm{~m}(\mathrm{R} 1)$, mostrado na Fig. 5. A geometria do SFI foi subtraída de R1 após criação de espessura, da mesma maneira foram atribuídas as condições de contorno apropriadas mantendo adiabáticas as superfícies que não fazem parte da cavidade. $\mathrm{O}$ processo é ilustrado na Fig. 6. As condições de contorno de Dirichlet para a cavidade, em vermelho na figura, foi $\mathrm{T}=0 \mathrm{~K}$ e as condições de Neumann, em azul, foram de garantir adiabaticidade.

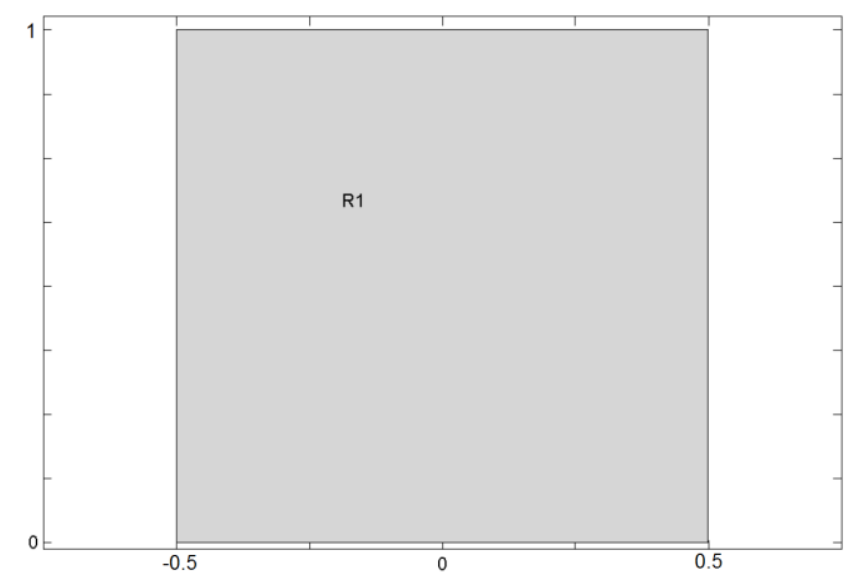

Figura 5: Domínio computacional inicial, de onde foram subtraídas as geometrias do SFI gerando as cavidades. 

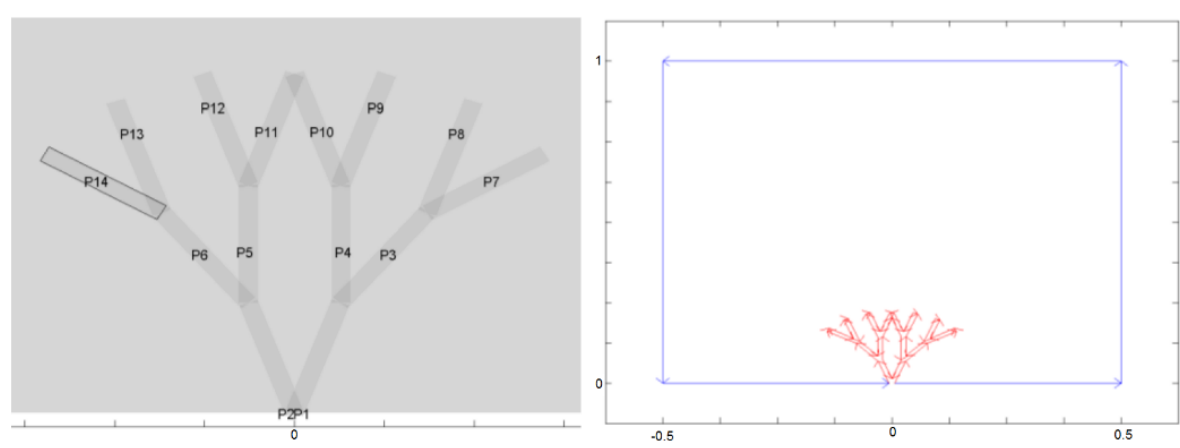

Figura 6: Exemplo de domínio computacional resultante no qual a equação do calor foi resolvida.

Em seguida foi gerada a malha para esse sistema e então a equação do calor foi resolvida. A equação é idêntica a Eq. 1, com os valores de q" e k iguais a unidade, entretanto qualquer valor positivo atribuído a essas constantes não alteraria as recomendações geométricas que foram obtidas. Conforme foi mostrado na análise de erro na seção anterior, a geração de malha do PDE TOOL é muito eficiente e por isso apenas dois refinamentos de malha foram executados antes de resolver a equação. A malha adaptativa gerada para a cavidade pelo PDE TOOL assim como a resolução da equação é mostrada a seguir na Fig. 7.
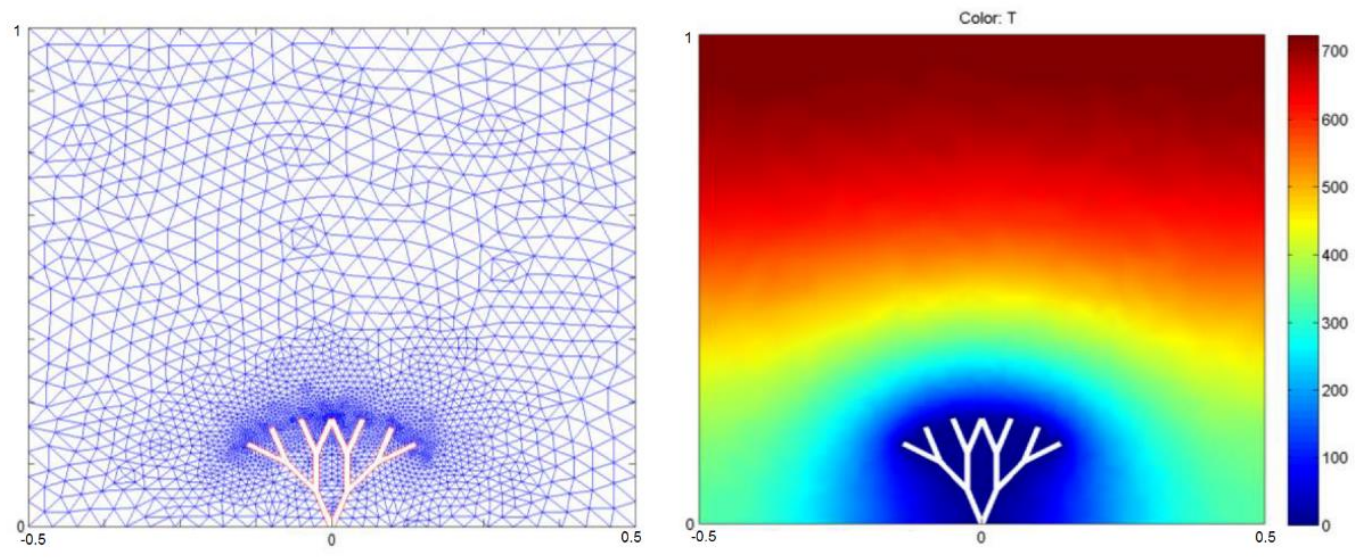

Figura 7: Cavidade com malha e resolução da EDP do calor para esse domínio.

Foi imposta uma restrição de área a esse problema de forma que a razão entre a área do domínio inicial $\left(A_{R 1}\right)$ e a área $A$ da cavidade $\left(A_{c}\right)$ fosse constante, nesse estudo foi mantido $\mathrm{A}_{\mathrm{R} 1} / \mathrm{A}_{\mathrm{c}}=0,01(1 \%)$. De forma a manter a restrição de área é necessário encontrar a raiz da seguinte equação:

$$
A_{c}(\delta)=0,01 \cdot A_{R 1}
$$

onde $\delta$ é a espessura dos braços da árvore. A forma da árvore não é uma geometria convencional de forma que não existe uma expressão analítica para $\mathrm{A}_{c}$, portanto a maneira mais eficiente de encontrar o $\delta$ apropriado é pela aplicação do método de Newton-Raphson, verificando se o erro na razão da área é menor que um determinado critério. Foi admitido nesse trabalho um erro de $10^{-5}$ na razão da área para obter o $\delta$.

Outro ponto que vale ressaltar é que algumas geometrias geradas pelo SFI podem acabar se estendendo além do domínio de R1, principalmente para braços muito longos ou ângulos muito abertos, essas geometrias foram descartadas dos resultados já que desviam da ideia de cavidade proposta. 


\subsection{OTIMIZAÇÃO GEOMÉTRICA}

O algoritmo foi implementado no ambiente MATLAB conforme ilustrado no fluxograma da Fig. 1 de forma que fosse possível testar diversos valores de $\theta,|v|$, e nI. O PDE TOOL calculou automaticamente os valores de $\mathrm{T}_{\max }$ para cada cavidade, o qual foi o critério de otimização escolhido. As geometrias com melhor desempenho, isto é, menor $\mathrm{T}_{\max }$ são consideradas mais eficientes pois dissipam melhor o calor. $\mathrm{O}$ efeito de cada uma das variáveis $\theta$, $|\mathrm{v}|$, e $\mathrm{nI}$ foram estudados nesse contexto. Um diagrama de árvore para as variáveis estudadas é mostrado a seguir:

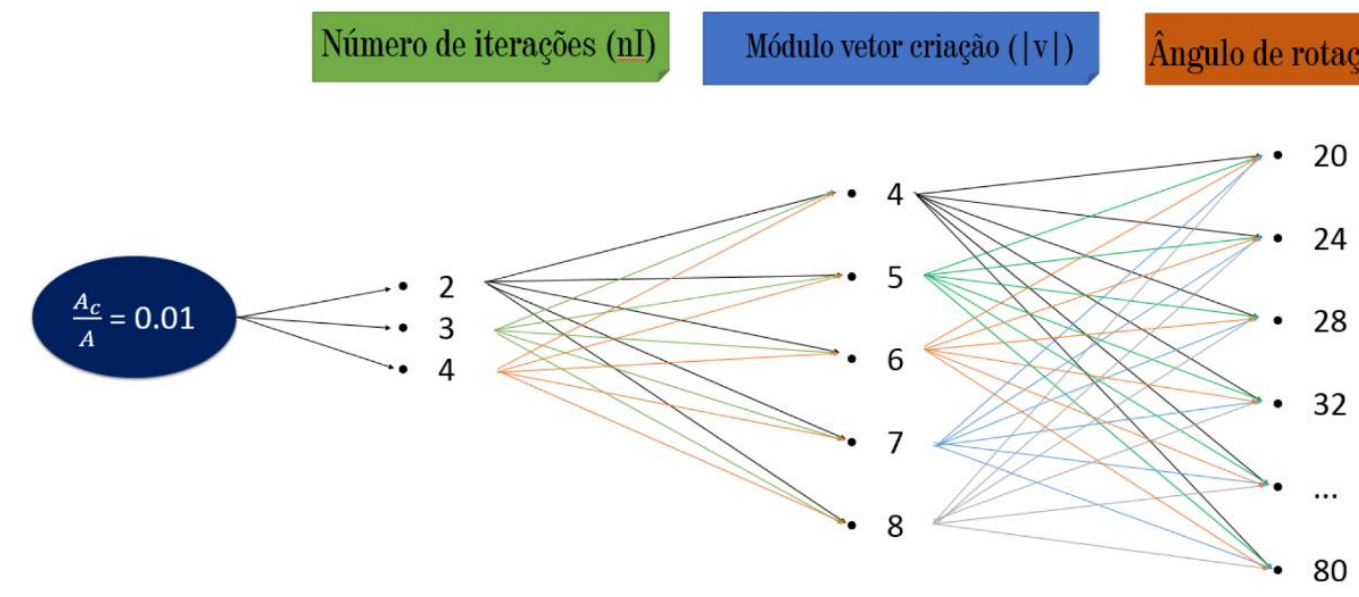

Figura 8: Diagrama de árvore para as simulações realizadas.

\section{RESULTADOS E DISCUSSÃO}

Em geral, quanto maior o número de iterações $(\mathrm{nI})$ e o tamanho dos braços das ramificações $(|\mathrm{v}|)$, melhor a dissipação do calor obtida. Porém a dependência do ângulo não seguiu um comportamento linear como podemos verificar esse comportamento nos gráficos da Fig. 9. A fim de determinar melhor o comportamento da dissipação do calor com o ângulo de bifurcação foi gerado um gráfico de cor para números de iteração fixo relacionando o ângulo e o módulo $|\mathrm{v}|$. Esse gráfico é mostrado para as 3 iterações testadas na Fig. 9. O gráfico mostra que ângulos de rotação entre $32^{\circ}$ e $46^{\circ}$ apresentam o melhor desempenho na redução de $T_{\max }$. No gráfico para $\mathrm{nI}=3$, observa-se que existem alguns valores faltando, esses valores foram desconsiderados pois nesse nível de bifurcação ângulos muito abertos ou braços muito longos fazem a cavidade ultrapassar o domínio inicial não formando mais uma cavidade.

O melhor resultado obtido foi para a geometria determinada pelos valores $n I=3, \theta=44^{\circ}$ e $|v|=8$. Essa geometria resulta em um $\mathrm{T}_{\max }$ de $270,45 \mathrm{~K}$. O campo de temperaturas para essa cavidade é mostrado na Fig. 10. Esses resultados preliminares indicam que aumentando o número de iterações e o tamanho dos braços deve levar a uma redução ainda maior da temperatura máxima do sistema. Também é interessante notar que aumentando o número de iterações um número de ângulos menor irá gerar uma cavidade efetiva de forma que um ângulo de convergência é esperado. Ainda não foi testado um algoritmo em que os braços de ramificação diminuem ou crescem com o número de iterações, isto é, em que o parâmetro de escala " $r$ " é diferente de 1, provavelmente uma redução maior da temperatura máxima do sistema será obtida nesse caso por causar um melhor preenchimento do espaço, uma vez que estaria mais de acordo com a ideia de facilitar o fluxo de calor conforme prevê o Design Construtal. 

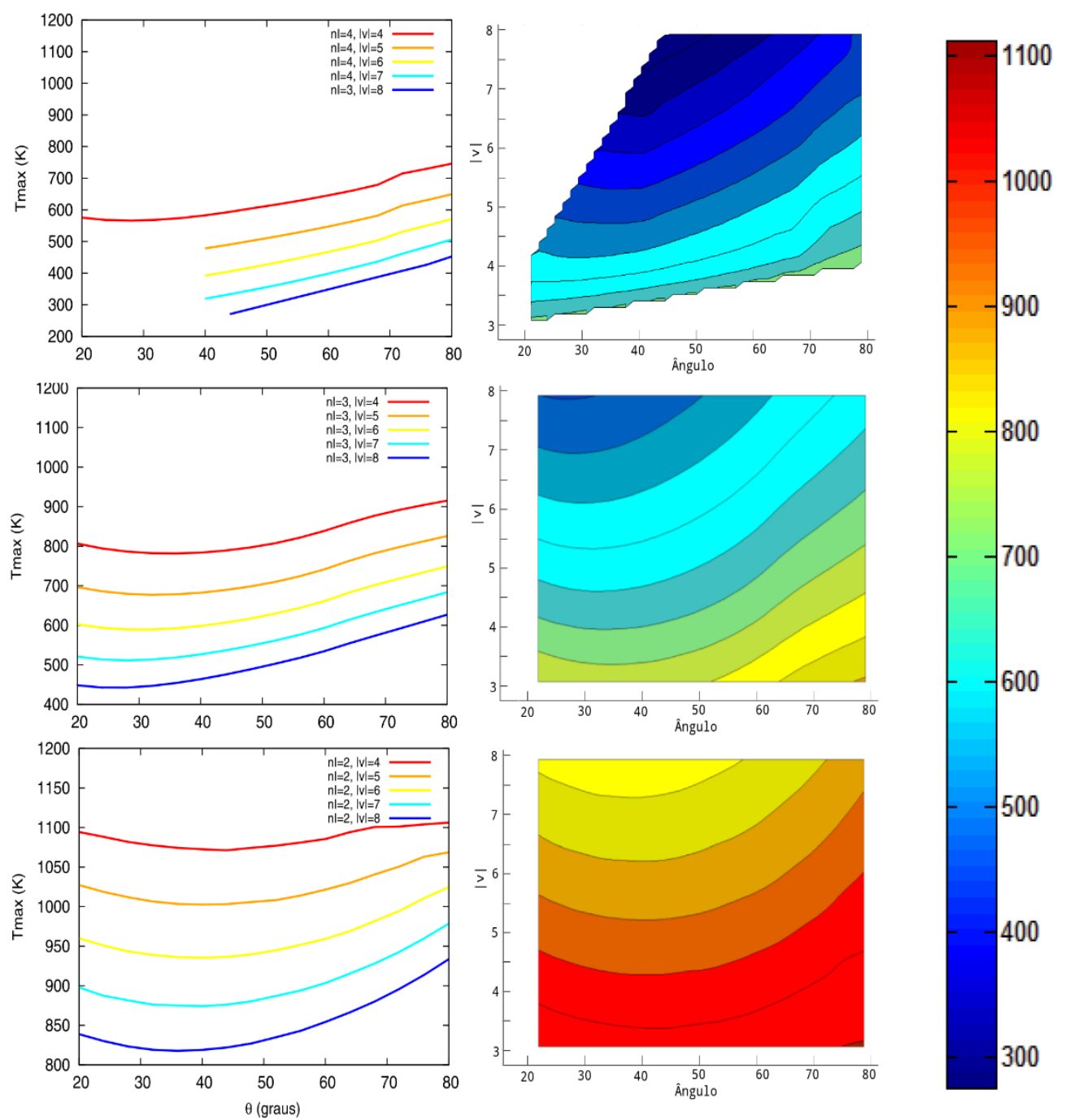

Figura 9: Gráfico interpolado do $T_{\max }$ em relação ao ângulo $(\theta)$ e $|v|$ para $n I=2,3,4$ de baixo para cima.

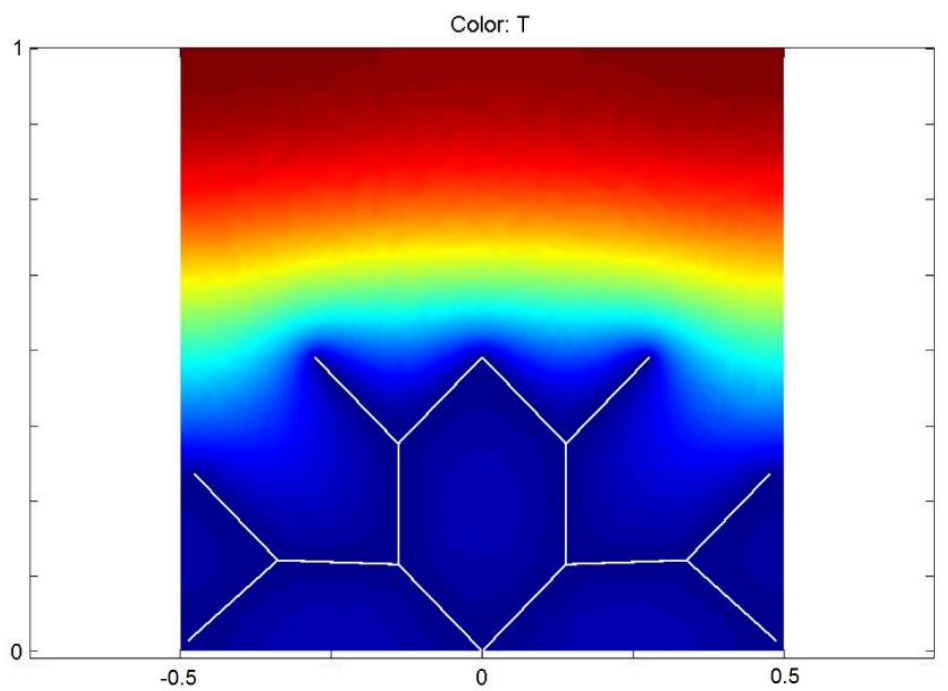

Figura 10: Mapa de temperaturas da cavidade ótima $\left(n I=3, \theta=44^{\circ}\right.$ e $\left.|v|=8\right)$ encontrada nesse trabalho. 


\section{CONCLUSÃO}

Neste trabalho a partir da ideia de usar geometria fractal, em particular, através de um sistema de funções iteradas, foi possível explorar com sucesso o espaço de busca de geometrias ótimas para dissipação de calor em uma cavidade. O procedimento aqui aplicado de alterar os parâmetros de geração de fractal ao invés de estudar as razões geométricas individualmente é mais geral e requer menos restrições para varrer o espaço de busca. Além disso, o uso de sistema de função iterada para criar uma cavidade multi-bifurcada está mais próximo da ideia construtal do projeto de que geometrias em forma de árvore facilitam o fluxo. Por esse motivo essa metodologia chega a resultados ótimos mais rapidamente do que usando geometrias fixas. Foi possível determinar uma cavidade ótima com parâmetros de geração $n I=3, \theta=44^{\circ}$ e $|v|=8$. Números de iterações maiores devem resultar em resultados ainda melhores de dissipação. O efeito do parâmetro de escala ' $r$ ' da função de iteração ainda deve ser estudado e provavelmente levará a um melhor preenchimento do domínio com melhor dissipação do calor.

\section{AGRADECIMENTOS}

Os autores agradecem ao Conselho Nacional de Desenvolvimento Científico e Tecnológico (CNPq) e Coordenação de Aperfeiçoamento de Pessoal de Nível Superior (CAPES) pelo financiamento para realização desse trabalho.

\section{REFERÊNCIAS BIBLIOGRÁFICAS}

1. Dekking M, Lévy-Véhel J, Lutton E, Tricot C. Fractals: Theory and Applications in Engineering: Theory and Applications in Engineering. Springer Science \& Business Media; 2012.

2. Vallejo LE, Lobo-Guerrero S, Chik Z. A network of fractal force chains and their effect in granular materials under compression. Fractals in Engineering. Springer; 2005. p. 67-80.

3. Bader R. Turbulent $\kappa-\varepsilon$ model of flute-like musical instrument sound production. Fractals in Engineering. Springer; 2005. p. 109-21.

4. Giona M, Cerbelli S, Adrover A. Invariant structures and multifractal measures in $2 \mathrm{~d}$ mixing systems. Fractals in Engineering. Springer; 2005. p. 141-55.

5. Cont R. Long range dependence in financial markets. Fractals in Engineering. Springer; 2005. p. 159-79.

6. Bertrand P. Financial modelling by multiscale fractional Brownian motion. Fractals in Engineering. Springer; 2005. p. 181-96.

7. Jones OD, Shen Y. A non-parametric test for self-similarity and stationarity in network traffic. Fractals in Engineering. Springer; 2005. p. 219-34.

8. Bona JL, Vrscay ER. Continuous evolution of functions and measures toward fixed points of contraction mappings. Fractals in Engineering. Springer; 2005. p. 237-53.

9. Nekka F, Li J. Various Mathematical Approaches to Extract Information from Textures of Increasing Complexities. Fractals in Engineering. Springer; 2005. p. 255-70.

10. Meneveau C, Katz J. Scale-invariance and turbulence models for large-eddy simulation. Annu Rev Fluid Mech. Annual Reviews 4139 El Camino Way, PO Box 10139, Palo Alto, CA 94303-0139, USA; 2000;32(1):1-32.

11. Sreenivasan K. Fractals and multifractals in fluid turbulence. Annu Rev Fluid Mech. Annual Reviews 4139 El Camino Way, PO Box 10139, Palo Alto, CA 94303-0139, USA; 1991;23(1):539604.

12. Ariza-Villaverde AB, Jiménez-Hornero FJ, De Ravé EG. Multifractal analysis of axial maps applied to the study of urban morphology. Comput Environ Urban Syst. Elsevier; 2013;38:1-10.

13. Krummel JR, Gardner RH, Sugihara G, O'neill R V, Coleman PR. Landscape patterns in a disturbed environment. Oikos. JSTOR; 1987;321-4.

14. Majumdar A, Bhushan B. Role of fractal geometry in roughness characterization and contact mechanics of surfaces. J Tribol. American Society of Mechanical Engineers; 1990;112(2):205

15. Stanley HE, Ostrowsky N. On growth and form: fractal and non-fractal patterns in physics. Springer Science \& Business Media; 2012.

16. Peitgen H-O, Jürgens H, Saupe D. Chaos and fractals: new frontiers of science. Springer Science \& Business Media; 2006. 
17. Bejan A, Lorente S. Design with constructal theory. 2008;

18. Lorenzini G, Biserni C. A Vapotron effect application for electronic equipment cooling. J Electron Packag. American Society of Mechanical Engineers; 2003;125(4):475-9.

19. Bejan A. Constructal-theory network of conducting paths for cooling a heat generating volume. Int $\mathbf{J}$ Heat Mass Transf. Elsevier; 1997;40(4):799-816.

20. Biserni C, Rocha LAO, Bejan A. Inverted fins: geometric optimization of the intrusion into a conducting wall. Int J Heat Mass Transf. Elsevier; 2004;47(12):2577-86.

21. Rocha LAO, Lorenzini E, Biserni C. Geometric optimization of shapes on the basis of Bejan's Constructal theory. Int Commun heat mass Transf. Elsevier; 2005;32(10):1281-8.

22. Xie Z, Chen L, Sun F. Geometry optimization of T-shaped cavities according to constructal theory. Math Comput Model. Elsevier; 2010;52(9):1538-46.

23. Biserni C, Rocha LAO, Stanescu G, Lorenzini E. Constructal H-shaped cavities according to Bejan's theory. Int J Heat Mass Transf. Elsevier; 2007;50(11):2132-8.

24. Lorenzini G, Garcia FL, dos Santos ED, Biserni C, Rocha LAO. Constructal design applied to the optimization of complex geometries: TY-shaped cavities with two additional lateral intrusions cooled by convection. Int J Heat Mass Transf. Elsevier; 2012;55(5):1505-12.

25. Bejan A, Lorente S. The constructal law of design and evolution in nature. Philos Trans R Soc B Biol Sci. The Royal Society; 2010;365(1545):1335-47.

26. MATLAB. version 6.0.088 (Release 12). Natick, Massachusetts: The MathWorks Inc.; 2000.

27. Reddy JN, Gartling DK. The finite element method in heat transfer and fluid dynamics. CRC press; 2010.

28. Zienkiewicz OC, Taylor RL. The finite element method for solid and structural mechanics. Butterworth-heinemann; 2005.

29. Cook RD, others. Concepts and applications of finite element analysis. John Wiley \& Sons; 2007.

30. Barnsley M, Hutchinson JE, Stenflo O. V-variable fractals and superfractals. arXiv Prepr math/0312314. 2003; 1 . 